\title{
Projection Effects and Strategic Ambiguity in Electoral Competition
}

Jensen, Thomas

Publication date:

2007

Document version

Publisher's PDF, also known as Version of record

Citation for published version (APA):

Jensen, T. (2007). Projection Effects and Strategic Ambiguity in Electoral Competition. Department of Economics, University of Copenhagen. 


\section{Discussion Papers Department of Economics University of Copenhagen}

\section{No. $07-12$ \\ Projection Effects and Strategic Ambiguity in Electoral Competition}

Thomas J ensen

Studiestræde 6, DK-1455 Copenhagen K., Denmark Tel. +45 35323082 - Fax +45 35323000 http://www.econ.ku.dk

ISSN: 0902-6452 (print) ISSN: 1601-2461 (online) 


\title{
Projection Effects and Strategic Ambiguity in Electoral Competition.
}

\author{
Thomas Jensen* \\ Department of Economics \\ University of Copenhagen
}

July 15, 2007

\begin{abstract}
Theories from psychology suggest that voters' perceptions of political positions depend on their non-policy related attitudes towards the candidates. A voter who likes (dislikes) a candidate will perceive the candidate's position as closer to (further from) his own than it really is. This is called projection. If voters' perceptions are not counterfactual and voting is based on perceived policy positions then projection gives a generally liked candidate an incentive to be ambiguous. In this paper we construct and analyze a formal model to investigate under which conditions this incentive survives in the strategic setting of electoral competition, even if voters dislike ambiguity per se.
\end{abstract}

Keywords: Electoral Competition, Ambiguity, Voter Perception, Cognitive Consistency, Projection.

JEL Classification: D72, D83.

* Department of Economics, University of Copenhagen, Studiestraede 6, DK-1455 Copenhagen K, Denmark. Email: Thomas.Jensen@econ.ku.dk. 


\section{Introduction}

According to theories from psychology (see e.g. Granberg (1993), Krosnick (2002), and references therein) people prefer to be in a state of cognitive consistency. Therefore a voter prefers to believe that he agrees with political candidates he likes (for non-policy related reasons) and disagrees with candidates he dislikes. One way the voter can achieve this is by distorting his perceptions of the candidates' policy positions. He "pulls" the positions of liked candidates towards his own position and "pushes" the positions of disliked candidates away from it. In general this is called projection. More specifically, positive projection ("pulling") is called assimilation and negative projection ("pushing") is called contrast.

If we assume that voters cannot have counterfactual perceptions then projection of a candidate's policy position can only happen when the candidate is ambiguous. So if projection effects exist and voters vote based on perceived policy positions then a generally liked candidate has an incentive to be ambiguous because of assimilation. This paper investigates under which conditions this incentive survives in the strategic setting of electoral competition.

We formulate and analyze an extension of the standard Downsian model that allows candidates to take ambiguous policy positions and introduces projection effects in voters' perceptions of such positions. Ambiguous positions are modelled by intervals of policies. Each voter has an (exogenous) positive, neutral or negative non-policy related attitude towards each candidate. When we say that a voter likes (dislikes) a candidate it simply means that he has a positive (negative) attitude towards him. Voters' perceptions of announced ambiguous positions are represented by probability distributions. They use perceived expected utility to decide on who to vote for. By assuming that voters are risk averse we get that they dislike ambiguity per se, i.e. if there were no projection effects. Thus any result predicting ambiguity is driven only by projection (assimilation).

Our first results answer the following question: Under which conditions can a candidate defeat the median by being ambiguous, i.e. win the election by taking an ambiguous position when the other candidate's position is fixed at the median? Loosely speaking, we show that if a candidate is liked by some voters and not disliked by too many then he can defeat the median. For example, a candidate who is not disliked by any voters can defeat the median if he is liked by an arbitrarily small group of voters.

Secondly, we consider the question of existence or non-existence of winning strategies (which must be ambiguous) for a candidate with an advantage due to voter attitudes and projection. Consider an advantaged candidate who is not disliked by any voters and liked by a majority and a disadvantaged candidate who is not liked by any voters. We show that the advantaged candidate has winning strategies if the assimilation effect is sufficiently strong. So our model does 
predict ambiguity in equilibrium when projection is strong. When projection is not sufficiently strong then the advantaged candidate does not have winning strategies and the model does not have an equilibrium (not even in mixed strategies). So the advantaged candidate may not be able to win the election for sure, it depends on the strength of the assimilation effect.

There is a large empirical literature on projection (see Granberg (1993) and Krosnick (2002) for surveys). A lot of studies using cross-sectional data do produce evidence that is consistent with projection effects. Krosnick (2002), however, points out that usually it is also consistent with alternative hypotheses, most notably policy based evaluation and persuation. A fairly recent study using crosssectional data is Merrill, Grofman and Adams (2001). Their findings are consistent with projection, but they also show that most of their evidence could be explained by policy based evaluation (together with different interpretations of issue scales among voters). Krosnick (2002) reviews a few panel data studies that carefully seek to separate projection from policy based evaluation and persuation. They do not find compelling evidence of projection. He concludes that the existence of projection has not yet been convincingly demonstrated and that further empirical research is needed.

As mentioned above, we make the assumption that projection of political positions is based on exogenous non-policy related attitudes and that the voting decision is policy based (given perceptions of positions). Thus we have a combination of projection and policy based evaluation and it seems hard to cast any verdict on its validity given the existing empirical literature on projection. Furthermore, the type of projection we consider is actually quite modest because we rule out counterfactual perceptions. So unless a candidate is very ambiguous voters' perceptions of his position will not be too inaccurate. Therefore our model can still be relevant even if voters' perceptions are reasonably accurate.

The paper is organized as follows. Section 2 contains a review of related literature. In Section 3 we set up the model and present some examples of our general model of projection of ambiguous policy positions. Section 4 contains our results (all proofs are delegated to the Appendix). In Section 5 we discuss and conlude.

\section{$2 \quad$ Related Literature}

A number of theoretical models of ambiguity in electoral competition exist in the literature. Zeckhauser (1969) and Fishburn (1972) both consider lotteries in social choice with a discrete set of alternatives (Zeckhauser only considers sets with three alternatives). They show that a lottery can never be a Condorcet winner.

Shepsle (1972) extends the standard Downsian model by forcing one of the candidates (the challenger) to take a lottery position, i.e. a non-degenerate proba- 
bility distribution over positions. The voters are expected utility maximizers. The main result is that if a majority of voters are risk loving on an interval containing the median, then the challenger can beat an incumbent at the median by taking a lottery position with mean equal to the median. However, both existence and non-existence of a winning position for the challenger can occur.

Page (1976) is critical of Shepsle's theory of ambiguity. He notes that the prediction of ambiguity is not very strong because the challenger may not have a winning strategy. Also he questions whether (a majority of) voters are really risk loving. Furthermore he argues that lottery positions are not a good way of modelling ambiguous political positions because candidates do not express their positions in ways that can easily be perceived as objective probability distributions. Page also presents his own theory of political ambiguity called emphasis allocation theory. He considers a multidimensional space of policy and valence dimensions. Candidates choose which dimensions (issues) to emphasize and take positions in these dimensions. They are vague/ambiguous on issues they do not put any emphasis on. Voters evaluate a candidate by summing the utilities of the candidate's positions on the issues, weigthed by the candidate's emphasis on each issue. In an example it is shown that this leads to emphasis on consensus issues and ambiguity on issues of conflict, no matter what the risk preferences of the voters are. In a footnote Page mentions that a possible different explanation of ambiguity could be that it allows for projection (p. 748).

McKelvey (1980) generalizes the results of Zeckhauser (1969) and Fishburn (1972) to continuous densities on $\mathbb{R}^{n}$. Furthermore he looks at the effect of introducing exogenous non-zero levels of ambiguity in a special case of electoral competition. He shows that it does not disrupt existing equilibria.

Glazer (1990) shows by some examples that risk loving voters are not necessary to get ambiguity in equilibrium in a two candidate electoral game. If there is uncertainty about the preferred policy of the candidates and the position of the median voter then ambiguity (not specifying a position) can be the equilibrium outcome of a model with simultaneous announcements. In a model with sequential announcements there can be ambiguity in equilibrium because the first mover does not want to make the second mover more informed about the position of the median voter.

Alesina and Cukierman (1990) considers a two period model. In the first period an incumbent decides on a policy. Before the second period elections are held and in the second period the winner enacts his preferred policy (final period play). Voters are imperfectly informed about the incumbents preferred policy so they vote using an estimate based on the first period policy. Therefore the incumbent can have an incentive to blur his policy preferences by being ambiguous, i.e. by choosing a noisy policy instrument. For a non-empty set of parameter values (including risk averse voters) it is optimal for the incumbent to choose a non-minimal level of 
ambiguity.

Aragones and Neeman (2000) analyze a two stage electoral game with two candidates. First the candidates simultaneously choose a point in the policy space (an ideology) and the choices become common knowledge. Then they simultaneously choose their level of ambiguity. Candidates have a preference for winning and for being ambiguous because it leaves them with more flexibility in office. Voters dislike ambiguity so candidates face a trade off between a high probability of winning (there is uncertainty about the position of the median voter) and being ambiguous. The main result is that when having flexibility in office is sufficiently important then there is policy divergence with a non-minimal level of ambiguity for both candidates. Otherwise there is policy convergence and no ambiguity.

In Aragones and Postlewaite (2002) a model with only three alternative policies and office motivated politicians is considered. Ambiguous positions are modelled as probability distributions on the set of alternatives. It is known from Fishburn (1972) that if candidates are not restricted in their choice of distributions then any equilibrium will consist of degenerate distributions. But Aragones and Postlewaite restrict the candidates such that each of them must put a minimum of probability mass on one of the alternatives - the alternative that voters think is most likely chosen by the candidate after the election. Under that assumption the result of Fishburn is not valid anymore. The most clean result is found when candidates are uncertain about the level of intensity of the voters preferences (then candidates payoff's are continuous). In that case it holds that (under some further assumptions) there always exists a pure strategy equilibrium and in any such equilibrium candidates are ambiguous (distributions are non-degenerate).

Meirowitz (2005) models a US presidential election with primaries. Each candidate can choose to announce a policy in the primaries or to be ambiguous in the primaries and not announce a policy until the general election. Candidates must stick to their policy announcements and they are imperfectly informed about voter preferences. In equilibrium candidates choose not to announce a policies in the primaries because it enables them to learn more about the electorate before committing to a policy position and because a candidate announcing a position in the primaries will be more vulnerable to an unconstrained opponent in the general election.

Our model is also related to the theoretical literature on valence advantage/candidate quality (see e.g. Ansolabehere and Snyder (2000), Groseclose (2001) and Aragones and Palfrey $(2002,2005))$. In these models one candidate has an advantage which makes all voters prefer him over the other candidate if there is policy convergence. In our model a candidate can have an advantage due to voters' attitudes. But he can only make use of that advantage by being ambiguous which makes voters who like him assimilate his position. Voters' attitudes does not directly influence their voting behavior. 


\section{The Model}

Our starting point is a standard one-dimensional spatial model with two candidates. We will extend that model by allowing candidates to take ambiguous policy positions and by introducing projection effects in voters' perceptions of such positions. In the following we describe the model in detail.

\subsection{The Candidates}

Before the election the two candidates announce policy positions. Each candidate can announce either a certain position or an ambiguous position. A certain position is represented by a point in the policy space $\mathbb{R}$. An ambiguous position is represented by a compact interval of policies. Thus the strategy space for each candidate can be written as

$$
S=\{[A-a, A+a] \mid A \in \mathbb{R}, a \geq 0\} .
$$

Announced positions are credible in the sense that the winning candidate must enact a policy in his announced interval. So certain positions are credible in the usual sense.

Each candidate's only objective is to win the election, none of them care about policy. Formally the preference relation of each candidate over the outcome of the election is given by

$$
\text { win } \succ \text { tie } \succ \text { loose. }
$$

Finally we assume that the candidates are fully informed about the electorate and that this is common knowledge.

\subsection{The Electorate}

There is a continuum of voters and each of them has a preferred point in the policy space $\mathbb{R}$. The distribution of preferred points is given by a density function $v$. We assume that $v$ is continuous and that the support of $v$ is an interval (bounded or unbounded). Without loss of generality we assume that the median voter is located at $x=0$, i.e.

$$
\int_{-\infty}^{0} v(x) d x=\int_{0}^{\infty} v(x) d x=\frac{1}{2} .
$$

Each voter has a utility function on the policy space. Let the utility function of the median voter be $u_{0}: \mathbb{R} \rightarrow \mathbb{R}$. Then the utility function $u_{x_{0}}$ of a voter with preferred point at $x_{0}$ is defined by

$$
u_{x_{0}}(x)=u_{0}\left(x-x_{0}\right) \text { for all } x \in \mathbb{R} .
$$


We assume that $u_{0}$ is symmetric around 0 , continuous on $\mathbb{R}$ and twice continuously differentiable on $\mathbb{R} \backslash\{0\}$ with

$$
\begin{aligned}
& u_{0}^{\prime}(x) \gtrless 0 \text { for } x \lessgtr 0, \\
& u_{0}^{\prime \prime}(x)<0 \text { for } x \neq 0 .
\end{aligned}
$$

Thus all voters are strictly risk averse.

We will now model how voters decide on which candidate to vote for. If each candidate announces a certain position then each voter simply votes for the candidate announcing the position with the highest utility. If at least one of the candidates announces an ambiguous position then it is less obvious how the voters should decide on who to vote for. We want them to use expected utility. But that is not straightforward since an ambiguous position is represented by an interval of policies rather than a probability distribution over policies. For a voter to use expected utility to evaluate an ambiguous position he has to somehow associate a probability distribution with the interval representing the position. The distribution represents the voter's perception of the ambiguous position. Or, to put it differently, the voter's belief about which policy the candidate will enact if elected. How voters perceive ambiguous positions is a crucial element of our model and we will use the rest of this section to describe it.

As mentioned in the introduction, the main idea is that a voter's perception of an ambiguous position depends on whether he has a positive, negative or neutral (non-policy related) attitude towards the candidate announcing it. If the voter likes the candidate, i.e. has a positive attitude towards him, then he will put most of the probability mass on the points of the interval that are closest to his preferred policy (assimilation). If the voter dislikes the candidate then he will do the opposite (contrast). And if the voter neither likes nor dislikes the candidate then he will spread the probability mass evenly across the interval. We will formalize this below.

For all voters the neutral perception of an ambiguous position is given by the uniform distribution on the interval. So the perceived expected utility of the ambiguous position $[A-a, A+a]$ for a neutral voter with preferred point $x_{0}$ is

$$
\frac{1}{2 a} \int_{A-a}^{A+a} u_{x_{0}}(x) d x .
$$

Since voters are strictly risk averse it follows that neutral voters dislike ambiguity.

To model assimilation perceptions first consider a voter with preferred point $x_{0} \geq 1$ and a positive attitude towards a candidate announcing $[-1,1]$. Thus we are modelling assimilation from the right of an ambiguous position centered at the median. The probability distribution that the voter associates with the 
ambiguous position is given by some cumulative distribution function $F_{1}^{1}$. So the voter's perceived expected utility of the candidate's position is

$$
\int_{-\infty}^{\infty} u_{x_{0}}(x) d F_{1}^{1}(x)
$$

We assume that $F_{1}^{1}$ satisfies the following two conditions. $U_{[-1,1]}$ denotes the cumulative distribution function of the uniform distribution on $[-1,1]$.

- $F_{1}^{1}$ puts no probability mass outside $[-1,1]$, i.e.

$$
F_{1}^{1}(x)=0 \text { for all } x<-1 \text { and } F_{1}^{1}(1)=1 .
$$

- $F_{1}^{1}$ strictly first order stochastically dominates the uniform distribution on $[-1,1]$, i.e.

$$
\begin{aligned}
F_{1}^{1}(x) & \leq U_{[-1,1]}(x) \text { for all } x \in \mathbb{R} \\
(\text { with } " & <\text { " for some } x \text { ). }
\end{aligned}
$$

The first condition says that there is no "counterfactual perception". Since the winning candidate must enact a policy in his announced interval the voter does not put any probability mass on policies outside the interval. The second condition is a convenient mathematical way of saying that the voter is assimilating the candidate's position from the right. It means that, for any $x \in[-1,1], F_{1}^{1}$ puts at least as much probability mass to the right of $x$ as the uniform distribution does (and strictly more for some $x$ ). Thus we see that, relative to the neutral perception given by the uniform distribution, the voter "pulls" probability mass to the right, i.e. towards his own preferred position.

We model assimilation of $[-1,1]$ from the left by symmetry. Therefore the assimilation perception for a voter with preferred point $x_{0} \leq-1$ is given by the distribution function $F_{1}^{-1}$ defined by

$$
F_{1}^{-1}(x)=1-\lim _{y \rightarrow(-x)^{-}} F_{1}^{1}(y) \text { for all } x \in \mathbb{R} .
$$

Because with this definition we have that, for any $x \in[-1,1], F_{1}^{-1}$ puts exactly as much probability mass on $[-1, x]$ as $F_{1}^{1}$ puts on $[-x, 1]$.

Then consider assimilation of $[-1,1]$ by some "interior voter", i.e. a voter with preferred point $x_{0} \in(-1,1)$. The distribution function representing the perception of such a voter is denoted $F_{1}^{x_{0}}$. Again we assume that

$$
F_{1}^{x_{0}}(x)=0 \text { for all } x<-1 \text { and } F_{1}^{x_{0}}(1)=1
$$


to rule out counterfactual perception. Formalizing the pulling of probability mass towards $x_{0}$ is a bit more tricky in this case than it was for "exterior voters", but the idea is the same. We want the distribution function to put more probability mass on points close to $x_{0}$ than the uniform distribution does. More precisely we assume that, for any $x>0, F_{1}^{x_{0}}$ puts at least as much probability mass on the interval $\left(x_{0}-x, x_{0}+x\right)$ as the uniform distribution does (and strictly more for some $x$ ). This can be written formally as

$$
\begin{aligned}
\lim _{y \rightarrow x^{-}} F_{1}^{x_{0}}\left(x_{0}+y\right)-F_{1}^{x_{0}}\left(x_{0}-x\right) & \geq U_{[-1,1]}\left(x_{0}+x\right)-U_{[-1,1]}\left(x_{0}-x\right) \text { for all } x>0 \\
(\text { with } " & >\text { "for some } x) .
\end{aligned}
$$

To have symmetry of perceptions we assume that, for any $x_{0} \in(-1,1)$,

$$
F_{1}^{-x_{0}}(x)=1-\lim _{y \rightarrow(-x)^{-}} F_{1}^{x_{0}}(y) \text { for all } x \in \mathbb{R} .
$$

Thus we are done modelling how voters assimilate the ambiguous position $[-1,1]$. Now we will extend the model to cover assimilation of all ambiguous strategies.

First consider assimilation of $[-a, a]$ for some $a>0$. In this case we use a simple scaling of the distribution functions defining assimilation of $[-1,1]$. More specifically the assimilation perceptions of voters with $x_{0} \geq 0$ are defined as follows (assuming symmetry this is all we need).

- For a voter with $x_{0} \geq a$ the distribution function is denoted $F_{a}^{a}$. It is defined by

$$
F_{a}^{a}(x)=F_{1}^{1}\left(\frac{x}{a}\right) \text { for all } x \in \mathbb{R} .
$$

- For a voter with $0 \leq x_{0}<a$ the distribution function is denoted $F_{a}^{x_{0}}$. It is defined by

$$
F_{a}^{x_{0}}(x)=F_{1}^{\frac{x_{0}}{a}}\left(\frac{x}{a}\right) \text { for all } x \in \mathbb{R} .
$$

It is easily seen that these distribution functions satisfy conditions that are analogous to the ones we imposed on the $F_{1}^{x_{0}}$ 's. Counterfactual perception is ruled out because we have, for any $0 \leq x_{0} \leq a$,

$$
F_{a}^{x_{0}}(x)=0 \text { for all } x<-a \text { and } F_{a}^{x_{0}}(a)=1 \text {. }
$$

And the $F_{a}^{x_{0}}$ 's are assimilation perceptions because

$$
\begin{aligned}
F_{a}^{a}(x) & \leq U_{[-a, a]}(x) \text { for all } x \in \mathbb{R} \\
\text { (with" } & <\text { " for some } x \text { ) }
\end{aligned}
$$


and, for any $0 \leq x_{0}<a$,

$$
\begin{aligned}
\lim _{y \rightarrow x^{-}} F_{a}^{x_{0}}\left(x_{0}+y\right)-F_{a}^{x_{0}}\left(x_{0}-x\right) & \geq U_{[-a, a]}\left(x_{0}+x\right)-U_{[-a, a]}\left(x_{0}-x\right) \text { for all } x>0 \\
(\text { with } " & >\text { for some } x) .
\end{aligned}
$$

Finally we will define assimilation of intervals of the type $[A-a, A+a]$ where $A \neq 0$. In that case we simply translate the distribution functions defining assimilation of $[-a, a]$ by the constant $A$. For example, the assimilation perception of $[A-a, A+a]$ by a voter with $x_{0} \geq A+a$ is given by the density function $F_{A, a}^{A+a}$ defined by

$$
F_{A, a}^{A+a}(x)=F_{a}^{a}(x-A) .
$$

Obviously the translated distribution functions satisfy the "translated" versions of the conditions satisfied by the $F_{a}^{x_{0}}$ 's.

Contrast perceptions are defined analogously to the way we have just defined assimilation perceptions. So, for example, the distribution function representing the contrast perception of voters to the right of some interval is strictly first order stochastically dominated by the uniform distribution on the interval. We use the same notation for contrast perceptions as for assimilation perceptions except that we replace the $F$ 's by $G$ 's. So the distribution functions representing contrast perception of $[-1,1]$ by voters to the right of the interval is denoted $G_{1}^{1}$ and so on.

For simplicity we assume that voters with the same preferred policy have the same attitude towards each candidate. Therefore we can define the attitude functions $L_{i}, i=1,2$, by

$$
L_{i}(x)=\left\{\begin{array}{r}
1 \text { if voters at } x \text { have a positive attitude towards Candidate } i \\
0 \text { if voters at } x \text { have a neutral attitude towards Candidate } i \\
-1 \text { if voters at } x \text { have a negative attitude towards Candidate } i
\end{array}\right\} .
$$

We make the technical assumption that, for each $i=1,2$, the sets $L_{i}^{-1}(\{1\})$, $L_{i}^{-1}(\{0\})$ and $L_{i}^{-1}(\{-1\})$ are Lebesgue measurable (such that we can integrate over them). Then the fraction of voters that have a positive/neutral/negative towards Candidate $i$ is

$$
\int_{L_{i}^{-1}(\{1\})} v(x) d x \quad / \quad \int_{L_{i}^{-1}(\{0\})} v(x) d x \quad / \quad \int_{L_{i}^{-1}(\{-1\})} v(x) d x .
$$

Before we move on we will give some examples of our model of assimilation. We will get back to these examples later on. 


\subsubsection{Example 1}

In our first example, the assimilation of $[-1,1]$ by voters to the right is given by the distribution function $F_{1}^{1}$ defined by

$$
F_{1}^{1}(x)=\left\{\begin{array}{cl}
\frac{1-\delta}{2} x+\frac{1-\delta}{2} & \text { if } x \in[-1,1) \\
1 & \text { if } x=1
\end{array}\right\},
$$

where $0<\delta<1$ is a parameter. This corresponds to a voter to the right believing that with probability $\delta$ the policy will be $x=1$ (the policy in the interval closest to his preferred point) and with probability $1-\delta$ the policy will be drawn from the uniform distribution on $[-1,1]$.

We want a voter with preferred point $x_{0} \in(-1,1)$ to have the same type of perception, i.e. to believe that with probability $\delta$ the policy will be $x=x_{0}$ and with probability $1-\delta$ the policy will be drawn from the uniform distribution. The distribution function corresponding to this perception is

$$
F_{1}^{x_{0}}(x)=\left\{\begin{array}{l}
\frac{1-\delta}{2} x-\frac{1-\delta}{2} \text { if } x \in\left[-1, x_{0}\right) \\
\frac{1-\delta}{2} x+\frac{1+\delta}{2} \text { if } x \in\left[x_{0}, 1\right]
\end{array}\right\} .
$$

It is easily seen that these distribution functions satisfy the required conditions. The example is extended to cover assimilation of all ambiguous positions as described above (by symmetry, scaling and translation).

\subsubsection{Example 2}

In our second example, the assimilation of $[-1,1]$ by voters to the right is given by the density function $f_{1}^{1}$ defined by

$$
f_{1}^{1}(x)=\left\{\begin{array}{c}
\frac{1-\gamma}{2} \text { if } x \in[-1,0) \\
\frac{1+\gamma}{2} \text { if } x \in[0,1]
\end{array}\right\},
$$

where $0<\gamma \leq 1$ is a parameter. 


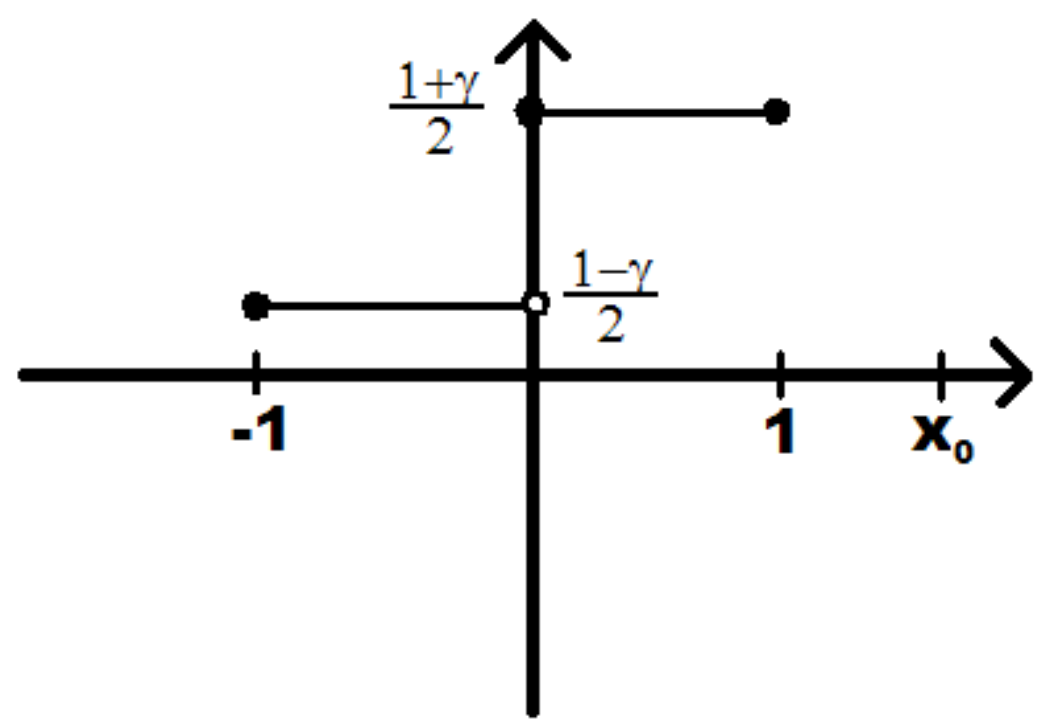

$$
f_{1}^{1} \text { for some } 0<\gamma<1 \text {. }
$$

We see how a voter to the right of the interval "pulls" probability mass towards his own preferred point. And we see that he does so without putting any probability mass on points outside the interval.

It is easily seen that the distribution function corresponding to the density function above is

$$
F_{1}^{1}(x)=\left\{\begin{array}{l}
\frac{1-\gamma}{2} x+\frac{1-\gamma}{2} \text { if } x \in[-1,0) \\
\frac{1+\gamma}{2} x+\frac{1-\gamma}{2} \text { if } x \in[0,1]
\end{array}\right\} .
$$

And then it is straightforward to show that the distribution strictly first order stochastically dominates the uniform distribution on $[-1,1]$.

The assimilation of $[-1,1]$ by voters with $x_{0} \in[0,1)$ is given by the density functions $f_{1}^{x_{0}}, x_{0} \in[0,1)$, defined by

$$
f_{1}^{x_{0}}=f_{1}^{1} \quad \text { if } \quad \frac{1}{2} \leq x_{0}<1
$$

and

$$
f_{1}^{x_{0}}(x)=\left\{\begin{array}{c}
\frac{1-\gamma}{2} \text { if }\left|x_{0}-x\right|>\frac{1}{2} \\
\frac{1+\gamma}{2} \text { if }\left|x_{0}-x\right| \leq \frac{1}{2}
\end{array}\right\} \quad \text { if } \quad 0 \leq x_{0}<\frac{1}{2}
$$




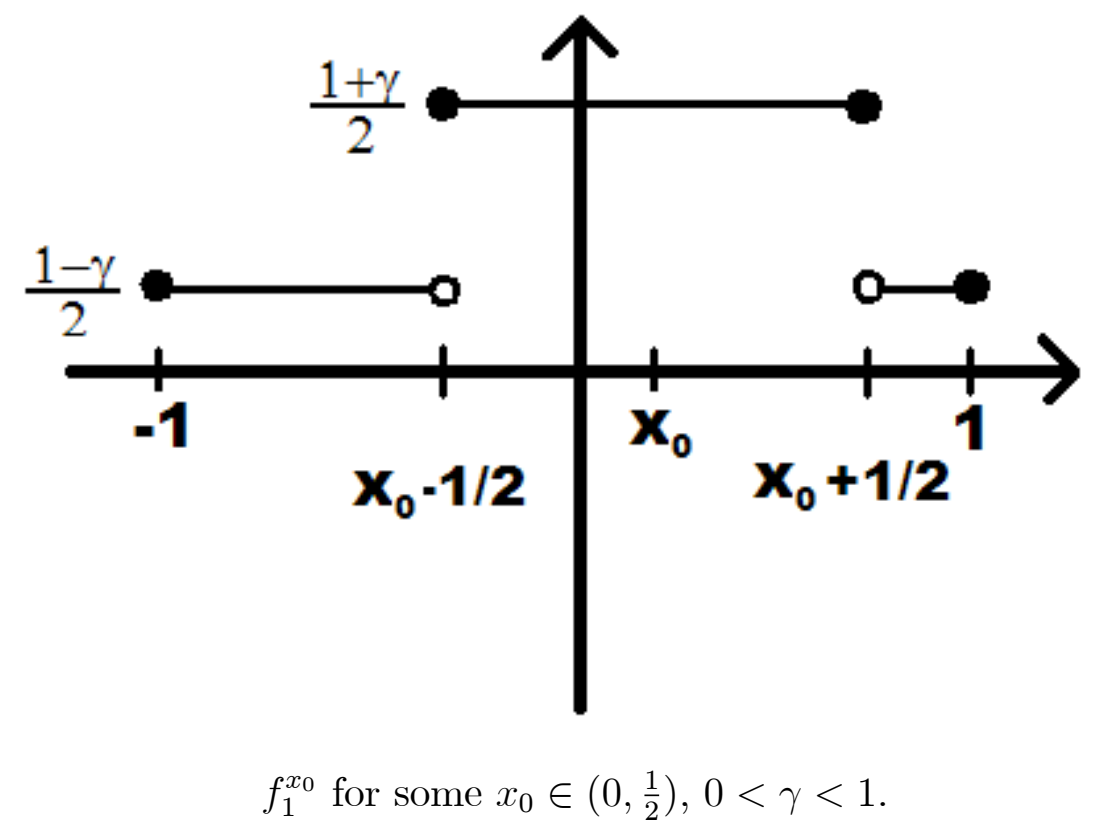

Given our definition of assimilation for "exterior voters" this is a natural way of defining it for "interior voters". In each case a voter puts a constant probability density of $\frac{1+\gamma}{2}$ on the half of the interval that is closest to his preferred point and a constant probability density of $\frac{1-\gamma}{2}$ on the rest.

It is straightforward to check that the distribution functions given by the $f_{1}^{x_{0}}$ 's satisfy the required conditions. As with our first example it is extended to cover assimilation of all ambiguous positions as described above.

\section{Results}

In this section we will address the questions in the list below. Our answers will help us understand how and why our introduction of ambiguous positions and projection effects changes the predictions of the standard model.

1. Under which conditions does the median voter theorem break down because candidates can take advantage of the assimilation effect by being ambiguous? More specifically, when can a candidate win the election by taking an ambiguous position when the other candidate's position is fixed at the median?

2. Under which conditions does a candidate with an advantage due to voters' non-policy related attitudes have a winning strategy? I.e. when does such a candidate have an ambiguous position that wins the election for him no matter what position the other candidate takes? 
3. What can we say about existence and properties of Nash equilibria?

All proofs are delegated to the Appendix.

\subsection{Defeating the Median}

Our first result shows that a candidate who is liked by a strict majority of voters can defeat the median, i.e. win the election when the other candidate's position is fixed at the median. Furthermore it shows that he can do so by being ambiguous around the median. Note that no additional assumptions on voter utility functions or voter perceptions are needed. The result holds even if voters are very risk averse and the assimilation effect is very small.

Theorem 4.1 Suppose Candidate $i$ is liked by a strict majority of voters, i.e.

$$
\int_{L_{i}^{-1}(\{1\})} v(x) d x>\frac{1}{2}
$$

Then there exists some $a^{\prime}>0$ such that, for any $0<a \leq a^{\prime}$, Candidate $i$ defeats the median by announcing the ambiguous position $[-a, a]$.

Being liked by a strict majority of voters is a necessary condition for a candidate to be able to defeat the median by an ambiguous position of the type $[-a, a]$, $a>0$. That follows immediately from the assumption that all voters are strictly risk averse. However, the following result shows that a candidate who is liked by less than a majority may be able to defeat the median by taking an ambiguous position that is not centrered at the median. The sets $X_{i}^{+}$and $X_{i}^{-}, i=1,2$, are defined by

$$
X_{i}^{+}=\left\{x \geq 0 \mid L_{i}(x)=0\right\} \cup\left\{x \mid L_{i}(x)=1\right\}
$$

and

$$
X_{i}^{-}=\left\{x \leq 0 \mid L_{i}(x)=0\right\} \cup\left\{x \mid L_{i}(x)=1\right\} .
$$

We let $E_{1}^{1}$ denote the expected value of $F_{1}^{1}$.

Theorem 4.2 Suppose $X_{i}^{+}$contains the preferred points of a strict majority of voters, i.e.

$$
\int_{X_{i}^{+}} v(x) d x>\frac{1}{2}
$$

Let $A \in\left(0, E_{1}^{1}\right)$. Then there exists some $a^{\prime}>0$ such that, for any $0<a \leq a^{\prime}$, Candidate $i$ defeats the median by announcing the ambiguous position

$$
[a A-a, a A+a]
$$


By symmetry it follows that if a strict majority of voters have preferred points in $X_{i}^{-}$and we let $A \in\left(-E_{1}^{1}, 0\right)$ then the same conlusion hold. Thus we see that a candidate who is liked by just a few voters may be able to defeat the median. For example that is the case if the candidate is not disliked by any voters.

The last result in this section shows that if neither $X_{i}^{+}$nor $X_{i}^{-}$contains the preferred points of a strict majority of voters then there exist voter perceptions (given by $F_{1}^{x_{0}}$ and $G_{1}^{x_{0}}, x_{0} \in[-1,1]$ ) such that Candidate $i$ cannot defeat the median. Thus we have a necessary and sufficient condition for Candidate $i$ to be able to defeat the median for any type of voter perceptions.

Theorem 4.3 Suppose that neither $X_{i}^{+}$nor $X_{i}^{-}$contains the preferred points of a strict majority of voters, i.e.

$$
\int_{X_{i}^{+}} v(x) d x \leq \frac{1}{2} \text { and } \int_{X_{i}^{-}} v(x) d x \leq \frac{1}{2} .
$$

Then there exist $F_{1}^{x_{0}}$ and $G_{1}^{x_{0}}, x_{0} \in[-1,1]$, (satisfying the assumptions on respectively assimilation and contrast perceptions) such that Candidate $i$ cannot defeat the median.

\subsection{Winning Strategies}

Our first observation is that Candidate $i$ does not have a winning strategy if the set of voters who like him $\left(L_{i}^{-1}(\{1\})\right)$ is not a strict majority. Because in that case Candidate $j$ can always get at least a tie by announcing the midpoint of Candidate $i$ 's position. So winning strategies can only exist if at least one candidate is liked by a strict majority of voters. Here we will only consider the case where one candidate (Candidate 1) is not disliked by any voters and liked by a strict majority and the other candidate (Candidate 2) is not liked by any voters. Thus we have

$$
L_{1}(x) \geq 0 \text { for all } x \in \mathbb{R}, \quad \int_{L_{i}^{-1}(\{1\})} v(x) d x>\frac{1}{2},
$$

and

$$
L_{2}(x) \leq 0 \text { for all } x \in \mathbb{R} .
$$

Assuming that Candidate 2 is not liked by any voters means that checking if some position of Candidate 1 is a winning strategy becomes a lot simpler. Because in that case we only need to check if Candidate 2 can get at least a tie against it by taking a certain position.

We will make some additional assumptions on voter utility functions and assimilation perceptions. We assume that voter utility functions are of the form

$$
u_{0}(x)=-|x|^{\alpha} \text { for some } \alpha>1 \text {. }
$$


With respect to assimilation perceptions we loosely speaking assume that if two voters have preferred points that are close then their assimilation perceptions are also close. More precisely we assume that for any sequence $\left(x_{n}\right)_{n \in \mathbb{N}}$ in $[-1,1]$ and any continuous function $f$ on $[-1,1]$,

$$
x_{n} \rightarrow x_{0} \Rightarrow \int_{-1}^{1} f(x) d F_{1}^{x_{n}}(x) \rightarrow \int_{-1}^{1} f(x) d F_{1}^{x_{0}}(x) .
$$

The following result gives conditions for existence and non-existence of winning strategies for Candidate 1 . Remember that $E_{1}^{1}$ denotes the expected value of $F_{1}^{1}$.

Theorem 4.4 With the additional assumptions from this subsection the following two statements hold.

1. Suppose there exists a $\beta>0$ such that

$$
(-\beta, \beta) \subseteq L_{1}^{-1}(\{1\})
$$

and

$$
\int_{-1}^{1} u_{x_{0}}(x) d F_{1}^{x_{0}}(x)>u_{x_{0}}\left(E_{1}^{1}\right) \text { for all }-1 \leq x_{0} \leq 0 .
$$

Then there exists an $a^{\prime}>0$ such that, for any $0<a \leq a^{\prime},[-a, a]$ is a winning strategy for Candidate 1.

2. Suppose

$$
u_{x_{0}}\left(E_{1}^{1}\right)>\int_{-1}^{1} u_{x_{0}}(x) d F_{1}^{x_{0}}(x) \text { for all } 0<x_{0}<1 .
$$

Then Candidate 1 does not have a winning strategy. More specifically, if Candidate 1 announces the ambiguous position $[A-a, A+a]$ then Candidate 2 can get at least a tie by announcing

$$
A-a E_{1}^{1} \text { if } A \geq 0
$$

and

$$
A+a E_{1}^{1} \text { if } A<0 .
$$

(And if Candidate 1 announces a certain position then Candidate 2 can get at least a draw by announcing the median.)

The first condition in the first statement says that Candidate 1 is liked by all voters in some neighborhood of the median. The second condition says that if a voter with $x_{0} \in[-1,0]$ likes Candidate 1 then he strictly prefers $[-1,1]$ announced by Candidate 1 over the certain position $E_{1}^{1}$. In the second statement the condition 
says that any voter with $x_{0}>0$ strictly prefers $E_{1}^{1}$ over $[-1,1]$ announced by Candidate 1.

The following lemma makes it easier to check for non-existence of winning strategies when some additional assumptions on assimilation perceptions are satisfied.

Lemma 4.5 Suppose that, for all $0<x_{0}<1$ and $x>0$,

$$
\lim _{y \rightarrow x^{-}} F_{1}^{0}(y)-F_{1}^{0}(-x) \geq \lim _{y \rightarrow x^{-}} F_{1}^{x_{0}}\left(x_{0}+y\right)-F_{1}^{x_{0}}\left(x_{0}-x\right) .
$$

Furthermore suppose that

$$
E_{1}^{x_{0}} \leq E_{1}^{1} \text { for all } 0<x_{0}<1 .
$$

Then the condition in part 2. of Theorem 4.4 is satisfied if

$$
\int_{-1}^{1} u_{0}(x) d F_{1}^{0}(x) \leq u_{0}\left(E_{1}^{1}\right)
$$

The new condition on the $F_{1}^{x_{0}}$ 's says that, for any $x>0, F_{1}^{0}$ puts at least as much probability mass on $(-x, x)$ as $F_{1}^{x_{0}}$ puts on $\left(x_{0}-x, x_{0}+x\right)$. It implies that the perceived expected utility of $[-1,1]$ for a voter at 0 with perception $F_{1}^{0}$ is at least as high as that for a voter at $x_{0}$ with perception $F_{1}^{x_{0}}$. The second condition says that the mean of the assimilation perception of $[-1,1]$ for voter a with $x_{0}<1$ is not higher than that for a voter with $x_{0} \geq 1$.

We will now use our results to analyze cases where assimilation perceptions are given by the two examples presented earlier.

\subsubsection{Example 1}

With this type of assimilation we have

$$
E_{1}^{1}=(1-\delta) \int_{-1}^{1} x d x+\delta=\delta
$$

It is straightforward to show that the additional assumptions from this subsection and the assumptions in Lemma 4.5 are satisfied. Thus we see that Candidate 1 does not have a winning strategy if

$$
\frac{1-\delta}{2} \int_{-1}^{1}-|x|^{\alpha} d x+\delta\left(-|0|^{\alpha}\right) \leq-|\delta|^{\alpha} .
$$

By straightforward calculations this inequality can be reduced to

$$
\delta^{\alpha}+\frac{\delta-1}{1+\alpha} \leq 0
$$


For any $\alpha>1$ the expression on the left hand side is negative for $\delta=0$, positive for $\delta=1$ and differentiable (w.r.t. $\delta$ ) on $[0,1]$ with positive derivative. Therefore there exists a $\delta^{*}(\alpha) \in(0,1)$ such that Candidate 1 does not have a winning strategy if $\delta \leq \delta^{*}(\alpha)$. We can calculate $\delta^{*}(2)$ explicitly by solving a second order equation. We get

$$
\delta^{*}(2)=\frac{\sqrt{13}-1}{6} \approx .43 .
$$

Our general results does not directly allow us to conclude that Candidate 1 has winning strategies if $\delta>\delta^{*}(\alpha)$. But we will now show that this is in fact the case (assuming that Candidate 1 is liked by all voters in some neighborhood of the median). For each $-1 \leq x_{0} \leq 0$ there exists a unique $C^{x_{0}}>x_{0}$ such that

$$
\int_{-1}^{1} u_{x_{0}}(x) d F_{1}^{x_{0}}(x)=u_{x_{0}}\left(C^{x_{0}}\right)
$$

(I.e. for a voter at $x_{0}, C^{x_{0}}$ is the certainty equivalent of $F_{1}^{x_{0}}$ to the right of $x_{0}$ ).

Lemma 4.6 For each $-1 \leq x_{0} \leq 0$ we have $C^{x_{0}} \leq C^{0}$.

It follows from the lemma that

$$
\int_{-1}^{1} u_{x_{0}}(x) d F_{1}^{x_{0}}(x)=u_{x_{0}}\left(C^{x_{0}}\right) \geq u_{x_{0}}\left(C^{0}\right) \text { for all }-1 \leq x_{0} \leq 0 .
$$

Since $\delta>\delta^{*}(\alpha)$ we have $u_{0}\left(C^{0}\right) \geq u_{0}\left(E_{1}^{1}\right)$ and thus $C^{0}<E_{1}^{1}$. Therefore we have

$$
u_{x_{0}}\left(C^{0}\right)>u_{x_{0}}\left(E_{1}^{1}\right) \text { for all }-1 \leq x_{0} \leq 0 .
$$

And then we can use Theorem 4.4 to conlude that Candidate 1 has winning strategies.

\subsubsection{Example 2}

With this type of assimilation we have

$$
\begin{aligned}
E_{1}^{1} & =\int_{-1}^{1} x f_{1}^{1}(x) d x \\
& =\frac{1-\gamma}{2} \int_{-1}^{-\frac{1}{2}} x d x+\frac{1+\gamma}{2} \int_{-\frac{1}{2}}^{\frac{1}{2}} x d x+\frac{1-\gamma}{2} \int_{\frac{1}{2}}^{1} x d x \\
& =\frac{\gamma}{2}
\end{aligned}
$$


Again it is straightforward to show that the additional assumptions from this subsection and the assumptions in Lemma 4.5 are satisfied. Thus we see that Candidate 1 does not have a winning strategy if

$$
\frac{1-\gamma}{2} \int_{-1}^{-\frac{1}{2}}-|x|^{\alpha} d x+\frac{1+\gamma}{2} \int_{-\frac{1}{2}}^{\frac{1}{2}}-|x|^{\alpha} d x+\frac{1-\gamma}{2} \int_{\frac{1}{2}}^{1}-|x|^{\alpha} d x \leq-\left|\frac{\gamma}{2}\right|^{\alpha}
$$

By straightforward calculations this inequality can be reduced to

$$
\left(\frac{\gamma}{2}\right)^{\alpha}+\frac{\gamma}{1+\alpha}\left(1-\left(\frac{1}{2}\right)^{\alpha}\right)-\frac{1}{1+\alpha} \leq 0
$$

For any $\alpha>1$ the expression on the left hand side is negative for $\gamma=0$, positive for $\gamma=1$ and differentiable (w.r.t. $\gamma$ ) on $[0,1]$ with positive derivative. Therefore there exists a $\gamma^{*}(\alpha) \in(0,1)$ such that Candidate 1 does not have a winning strategy if $\gamma \leq \gamma^{*}(\alpha)$. We can calculate $\gamma^{*}(2)$ explicitly by solving a second order equation. We get

$$
\gamma^{*}(2)=\frac{\sqrt{57}-3}{6} \approx .76 .
$$

Our general results does not directly allow us to conclude that Candidate 1 has winning strategies if $\gamma>\gamma^{*}(\alpha)$. But we will now show that this is in fact the case (assuming that Candidate 1 is liked by all voters in some neighborhood of the median). As in Example 1 it suffices to show that $C^{x_{0}} \leq C^{0}$ for all $-1 \leq x_{0} \leq 0$. That follows from the two lemmas below.

Lemma 4.7 $C^{x_{0}} \leq C^{0}$ for all $-\frac{1}{2} \leq x_{0} \leq 0$.

Lemma $4.8 C^{x_{0}} \leq C^{-\frac{1}{2}}$ for all $-1 \leq x_{0} \leq-\frac{1}{2}$.

\subsection{Nash Equilibria}

\subsubsection{Pure Strategies}

Here we will make some observations about the existence and properties of pure strategy Nash equilibria.

First, consider the case where neither candidate can defeat the median. Then $\left(s_{1}^{*}, s_{2}^{*}\right)=(0,0)$, i.e. convergence to the median, is an equilibrium because neither candidate can win the election by deviating to another position. Furthermore, if neither of the candidates are liked by exactly $50 \%$ of the voters then $(0,0)$ is the unique equilibrium (each candidate can then defeat any position different from the median). Thus the median voter theorem holds in this situation. If a candidate is liked by exactly $50 \%$ of the voters (and thus disliked by the other $50 \%$ 
- otherwise he could defeat the median) then there could exist equilibria where he is ambiguous. But the outcome would always be a tie and he could also get a tie by deviating to the median.

Secondly, consider the case where one candidate (Candidate 1) can defeat the median but the other (Candidate 2) cannot. If Candidate 2 is liked by strictly less than $50 \%$ of the voters then Candidate 1 can defeat any position of Candidate 2. Therefore we have that in any equilibrium Candidate 1 must win the election. Thus $\left(s_{1}^{*}, s_{2}^{*}\right)$ is an equilibrium if and only if $s_{1}^{*}$ is a winning strategy for Candidate 1 (which must be ambiguous). So we have existence of Nash equilibria if and only if we have existence of winning strategies for Candidate 1. If Candidate 2 is liked by exactly $50 \%$ of the voters then the equilibrium outcome is a tie if Candidate 2 has a position that gives him at least a tie against any position of Candidate 1. If Candidate 2 does not have such a position then we again have that in any equilibrium Candidate 1 must win the election. In both situations it follows that in any equilibrium at least one candidate will be ambiguous.

Finally, consider the case where both candidates can defeat the median. Without loss of generality assume that Candidate 1 is liked by at least as many voters as Candidate 2. If Candidate 2 is liked by strictly less than $50 \%$ of the voters then we have that $\left(s_{1}^{*}, s_{2}^{*}\right)$ is an equilibrium if and only if $s_{1}^{*}$ is a winning strategy for Candidate 1 (Candidate 1 can defeat any position of Candidate 2). If Candidate 2 is liked by at least $50 \%$ of the voters then consider the numbers

$$
P_{i}=\int_{\left\{x \mid L_{i}(x)>L_{j}(x)\right\}} v(x) d x, \quad i=1,2, \quad j \neq i .
$$

$P_{i}$ is the share of voters who has a more positive attitude towards Candidate $i$ than towards Candidate $j$. If $P_{i}>P_{j}$ then Candidate $i$ can defeat any position of Candidate $j$ by imitation. Thus it follows that $\left(s_{1}^{*}, s_{2}^{*}\right)$ is an equilibrium if and only if $s_{i}^{*}$ is a winning strategy for Candidate $i$. If $P_{i}=P_{j}$ then each candidate can get a tie against any position of the other candidate (again by imitation). Thus any equilibrium outcome must be a tie and both candidates must be ambiguous in equilibrium.

\subsubsection{Mixed Strategies}

We have just seen that when at least one candidate can defeat the median, then, except perhaps for some very special cases, pure strategy Nash equilibria only exist if one candidate has a winning strategy. And we know from earlier that winning strategies do not always exist even in the extreme case where one candidate is liked by all voters and the other candidate is disliked by all voters. So it is natural to ask the question if mixed strategy equilibria exist when pure strategy equilibria do not. 
Consider again the model with the extra assumptions from the subsection on winning strategies. The question then is if there exists a mixed strategy equilibrium when Candidate 1 does not have a winning strategy. The result below shows that when the condition for non-existence of winning strategies in Theorem 4.4 is satisfied then the answer is no (except perhaps in a knife edge case).

Theorem 4.9 Suppose all the assumptions from the subsection on winning strategies are satisfied. If

$$
u_{x_{0}}\left(E_{1}^{1}\right)>\int_{-1}^{1} u_{x_{0}}(x) d F_{1}^{x_{0}}(x) \text { for all } 0 \leq x_{0}<1
$$

then there does not exist a mixed strategy Nash equilibrium.

With respect to our examples of assimilation, the theorem implies that if $\delta<\delta^{*}(\alpha)$ (in Example 1) or $\gamma<\gamma^{*}(\alpha)$ (in Example 2) then a mixed strategy equilibrium does not exist.

\section{Discussion}

Our goal in this paper has been to theoretically investigate if positive projection (assimilation) of policy positions can explain why some politicians are ambiguous with respect to their issue positions. To do that we have extended the standard Downsian model of electoral competition by allowing candidates to take ambiguous policy positions and by introducing projection effects in voters' perceptions of such positions. By assuming that voters dislike ambiguity per se (if attitudes are neutral then voters dislike ambiguity because of risk aversion) we have made sure that projection is the driving force behind any result predicting ambiguity.

In the standard model the median defeats any other position. Therefore a natural first step was to find out under which conditions a candidate can defeat the median by being ambiguous. We presented necessary and sufficient conditions. We saw that it may suffice for a candidate to be liked by only a small group of voters as long as he is not disliked by too many. For example, if a candidate is not disliked by any voters then he can defeat the median if he is liked by an arbitrarily small group of voters.

While having at least one candidate that is able to defeat the median by being ambiguous is certainly a necessary condition for predicting ambiguity it is far from sufficient. Electoral competition is a strategic situation and there is no reason to assume that one candidate will announce the median if he can do better by taking a different position. Therefore our next step was to look at existence and non-existence of winning strategies (which must be ambiguous). We restricted 
attention to the case where one candidate is not liked by any voters and made some additional assumptions on voter utility functions and perceptions. We saw that if the assimilation effect is sufficiently strong then a candidate who is not disliked by any voters and liked by a strict majority has winning strategies. But even if he is liked by all voters he may not have winning strategies. So our model does predict that a generally liked candidate will be ambiguous if he is running against a candidate who is generally not liked and the assimilation effect is sufficiently strong. But even a candidate with the largest possible advantage due to voters' non-policy related attitudes may not have a position that wins the election for him (if the assimilation effect is not strong enough). That is a rather striking result.

While the possible non-existence of winning strategies even for a candidate with the largest possible advantage due to voters' attitudes is an interesting feature of the model it is also problematic. Because, as our observations on Nash equilibria revealed, when at least one candidate can defeat the median then non-existence of winning strategies implies non-existence of pure strategy equilibria (except perhaps in some very special cases). So when the median voter theorem breaks down but neither candidate has a winning strategy then our model does not give us clear predictions in terms of pure strategy equilibria. And we have also seen that turning attention to mixed strategy equilibria is not a solution to this problem. So an obvious direction for further research is to come up with a model that give better equilibrium predictions.

\section{References}

1. Alesina, A. and Cukierman, A. (1990). The Politics of Ambiguity, The Quarterly Journal of Economics 105: 829-850.

2. Ansolabehere, S. D. and Snyder, Jr., J. M. (2000). Valence Politics and Equilibrium in Spatial Election Models, Public Choice 103: 327-336.

3. Aragonès, E. and Neeman, Z. (2000). Strategic Ambiguity in Electoral Competition, Journal of Theoretical Politics 12: 183-204.

4. Aragonès, E. and Palfrey, T. R. (2002). Mixed Equilibrium in a Downsian Model with a Favored Candidate, Journal of Economic Theory 103: 131-161.

5. Aragonès, E. and Palfrey, T. R. (2005). Electoral Competition Between Two Candidates of Different Quality: The Effects of Candidate Ideology and Private Information. In D. Austen-Smith and J. Duggan (Editors), Social Choice and Strategic Decisions: Essays in Honor of Jeffrey S. Banks (pp. 93-112). Springer. 
6. Aragonès, E. and Postlewaite, A. (2002). Ambiguity in Election Games, Review of Economic Design 7: 233-255.

7. Fishburn, P. C. (1972). Lotteries and Social Choice, Journal of Economic Theory 5: 189-207.

8. Glazer, A. (1990). The Strategy of Candidate Ambiguity, The American Political Science Review 84: 237-241.

9. Granberg, D. (1993). Political Perception. In S. Iyengar and W. J. McGuire (Editors), Explorations in Political Psychology (pp. 70-112). Duke University Press.

10. Groseclose, T. J. (2001). A Model of Candidate Location When One Candidate Has a Valence Advantage, American Journal of Political Science 45: 862-86.

11. Krosnick, J. A. (2002). The Challenges of Political Psychology: Lessons to Be Learned from Research on Attitude Perception. In J. Kuklinski (Editor), Thinking about Political Psychology (pp. 115-152). Cambridge University Press.

12. McKelvey, R. D. (1980). Ambiguity in Spatial Models of Policy Formation, Public Choice 35: 385-402.

13. Meirowitz, A. (2005). Informational Party Primaries and Strategic Ambiguity, Journal of Theoretical Politics 17(1): 107-136.

14. Merrill, III, S., Grofman, B. and Adams, J. (2001). Assimilation and Contrast Effects in Voter Projections of Party Locations: Evidence from Norway, France and the USA, European Journal of Political Research 40: 199-221.

15. Page, B. (1976). The Theory of Political Ambiguity, The American Political Science Review 70: 742-752.

16. Shepsle, K. (1972). The Strategy of Ambiguity: Uncertainty and Electoral Competition, The American Political Science Review 66: 555-568.

17. Zeckhauser, R. (1969). Majority Rule with Lotteries on Alternatives, The Quarterly Journal of Economics 83: 696-703. 


\section{Appendix}

Proof of Theorem 4.1.

For each $n \in \mathbb{N}$ let

$$
X_{i}^{n}=\left\{x\left|L_{i}(x)=1, \frac{1}{n} \leq\right| x \mid \leq n\right\} .
$$

Since a strict majority of voters likes Candidate $i$ there exists an $N \in \mathbb{N}$ such that $X_{i}^{N}$ contains the preferred points of a strict majority of voters. We will prove that there exists an $a^{\prime}>0$ such that, for any $0<a \leq a^{\prime}$, all voters with $x_{0} \in X_{i}^{N}$ strictly prefer $[-a, a]$ announced by Candidate $i$ over the median $(x=0)$.

For each $a>0$,

$$
\max _{-a \leq y \leq a}\left|u_{x_{0}}^{\prime \prime}(y)\right|
$$

is a continuous function of $x_{0}$ on $(a, \infty)$. So for $a<\frac{1}{N}$ it follows by compactness that the function is bounded on $\left[\frac{1}{N}, N\right]$. Thus we can define

$$
C_{a}=\max _{\frac{1}{N} \leq x_{0} \leq N} \max _{-a \leq y \leq a}\left|u_{x_{0}}^{\prime \prime}(y)\right| .
$$

Now let $\frac{1}{N} \leq x_{0} \leq N, 0<a<\frac{1}{N}$ and $x \in[-a, a]$. Then, by Taylor's theorem, we have

$$
u_{x_{0}}(x)=u_{x_{0}}(0)+u_{x_{0}}^{\prime}(0) x+\frac{u_{x_{0}}^{\prime \prime}(\xi)}{2} x^{2} .
$$

for some $\xi \in[-a, a]$ (actually between 0 and $x$ ). And thus it follows that

$$
u_{x_{0}}(x) \geq u_{x_{0}}(0)+u_{x_{0}}^{\prime}(0) x-\frac{C_{a}}{2} x^{2} .
$$

Using this inequality we get $\left(E_{1}^{1}\right.$ denotes the expected value of $\left.F_{1}^{1}\right)$

$$
\begin{aligned}
\int_{-a}^{a} u_{x_{0}}(x) d F_{a}^{a}(x) & \geq \int_{-a}^{a}\left(u_{x_{0}}(0)+u_{x_{0}}^{\prime}(0) x-\frac{C_{a}}{2} x^{2}\right) d F_{a}^{a}(x) \\
& =u_{x_{0}}(0)+u_{x_{0}}^{\prime}(0) \int_{-a}^{a} x d F_{a}^{a}(x)-\frac{C_{a}}{2} \int_{-a}^{a} x^{2} d F_{a}^{a}(x) \\
& =u_{x_{0}}(0)+u_{x_{0}}^{\prime}(0) a E_{1}^{1}-\frac{C_{a}}{2} \int_{-a}^{a} x^{2} d F_{a}^{a}(x) \\
& \geq u_{x_{0}}(0)+u_{x_{0}}^{\prime}(0) a E_{1}^{1}-\frac{C_{a}}{2} a^{2} \int_{-a}^{a} d F_{a}^{a}(x) \\
& =u_{x_{0}}(0)+u_{x_{0}}^{\prime}(0) a E_{1}^{1}-\frac{C_{a}}{2} a^{2} \\
& \geq u_{x_{0}}(0)+u_{0}^{\prime}\left(-\frac{1}{N}\right) a E_{1}^{1}-\frac{C_{a}}{2} a^{2}
\end{aligned}
$$


Since $u_{0}^{\prime}\left(-\frac{1}{N}\right)>0, E_{1}^{1}>0$ and $C_{a}$ is decreasing with $a$ it follows that, for $a$ sufficiently small,

$$
\int_{-a}^{a} u_{x_{0}}(x) d F_{a}^{a}(x)>u_{x_{0}}(0) \text { for all } \frac{1}{N} \leq x_{0} \leq N
$$

So all voters with $\frac{1}{N} \leq x_{0} \leq N$ and $L_{i}\left(x_{0}\right)=1$ strictly prefer $[-a, a]$ announced by Candidate $i$ over the median for $a$ sufficiently small. By symmetry the same holds for voters with $-N \leq x_{0} \leq-\frac{1}{N}$ and $L_{i}\left(x_{0}\right)=1$. Thus it holds for all voters with $x_{0} \in X_{i}^{N}$.

Proof of Theorem 4.2.

Let $A \in\left(0, E_{1}^{1}\right)$. Pick an $N \in \mathbb{N}$ such that a majority of voters have preferred points in

$$
X_{i}^{N+}=\left\{x\left|x \in X_{i}^{+}, \frac{1}{N} \leq\right| x \mid \leq N\right\} .
$$

For $a>0$ with $a A+a<\frac{1}{N}$ we can define

$$
C_{A, a}=\max _{\frac{1}{N} \leq\left|x_{0}\right| \leq N} \max _{a A-a \leq y \leq a A+a}\left|u_{x_{0}}^{\prime \prime}(y)\right| .
$$

Then, by Taylor's theorem, we get that for all $x_{0}$ with $\frac{1}{N} \leq\left|x_{0}\right| \leq N$ and all $a>0$ with $a A+a<\frac{1}{N}$

$$
u_{x_{0}}(x) \geq u_{x_{0}}(0)+u_{x_{0}}^{\prime}(0) x-\frac{C_{A, a}}{2} x^{2} \quad \text { for all } \quad x \in[a A-a, a A+a] .
$$

For all voters with $x_{0} \in X_{i}^{+}, \frac{1}{N} \leq x_{0} \leq N$ the perceived expected utility of $[a A-a, a A+a]$ is at least

$$
\frac{1}{2 a} \int_{a A-a}^{a A+a} u_{x_{0}}(x) d x .
$$

Using the inequality above we get that, for all $a>0$ with $a A+a<\frac{1}{N}$,

$$
\begin{aligned}
\frac{1}{2 a} \int_{a A-a}^{a A+a} u_{x_{0}}(x) d x & \geq \int_{a A-a}^{a A+a}\left(u_{x_{0}}(0)+u_{x_{0}}^{\prime}(0) x-\frac{C_{A, a}}{2} x^{2}\right) d x \\
& =u_{x_{0}}(0)+u_{x_{0}}^{\prime}(0) a A-\frac{C_{A, a}}{2} \int_{a A-a}^{a A+a} x^{2} d x \\
& \geq u_{x_{0}}(0)+u_{x_{0}}^{\prime}(0) a A-\frac{C_{A, a}}{2}(a A+a)^{2} \\
& \geq u_{x_{0}}(0)+u_{0}^{\prime}\left(-\frac{1}{N}\right) A a-\frac{C_{A, a}}{2}(1+A)^{2} a^{2} .
\end{aligned}
$$


The last expression is strictly greater than $u_{x_{0}}(0)$ for small $a>0$. So it follows that for $a$ sufficiently small all voters with $x_{0} \in X_{i}^{+}, \frac{1}{N} \leq x_{0} \leq N$ strictly prefer $[a A-a, a A+a]$ over the median.

For all voters with $x_{0} \in X_{i}^{+},-N \leq x_{0} \leq-\frac{1}{N}$ the perceived expected utility of $[a A-a, a A+a]\left(\right.$ with $\left.-\frac{1}{N}<a A-a\right)$ is

$$
\int_{a A-a}^{a A+a} u_{x_{0}}(x) d F_{a A, a}^{a A-a}(x)
$$

Using the "Taylor inequality" from above we get that, for all $a>0$ with $a A+a<\frac{1}{N}$,

$$
\begin{aligned}
\int_{a A-a}^{a A+a} u_{x_{0}}(x) d F_{a A, a}^{a A-a}(x) & \geq \int_{a A-a}^{a A+a}\left(u_{x_{0}}(0)+u_{x_{0}}^{\prime}(0) x-\frac{C_{A, a}}{2} x^{2}\right) d F_{a A, a}^{a A-a}(x) \\
& \geq u_{x_{0}}(0)+u_{x_{0}}^{\prime}(0)\left(a A-a E_{1}^{1}\right)-\frac{C_{A, a}}{2}(a A+a)^{2} \\
& \geq u_{x_{0}}(0)+u_{0}^{\prime}\left(\frac{1}{N}\right)\left(A-E_{1}^{1}\right) a-\frac{C_{A, a}}{2}(1+A)^{2} a^{2} .
\end{aligned}
$$

The last expression is is strictly greater than $u_{x_{0}}(0)$ for small $a>0$. So it follows that for $a$ sufficiently small all voters with $x_{0} \in X_{i}^{+},-N \leq x_{0} \leq-\frac{1}{N}$ strictly prefer $[a A-a, a A+a]$ over the median.

Thus we have seen that for $a>0$ sufficiently small, all voters with $x_{0} \in X_{i}^{N+}$ (a majority) prefer $[a A-a, a A+a]$ over the median.

\section{Proof of Theorem 4.3.}

Suppose that assimilation perceptions are as in Example 1 (for some $0<\delta<1$ ). Let contrast perceptions be given by

$$
G_{1}^{x_{0}}=\left\{\begin{array}{c}
F_{1}^{1} \text { if } x_{0} \in[-1,0) \\
F_{1}^{-1} \text { if } x_{0} \in[0,1]
\end{array}\right\} .
$$

Suppose Candidate $i$ announces some interval. We will show that at least $50 \%$ of the voters strictly prefer the median over the interval.

The interval can be written as

$$
[a A-a, a A+a] \text { for some } A \in \mathbb{R}, a>0 \text {. }
$$

For $A=0$ all voters with $L_{i}\left(x_{0}\right) \leq 0$ (at least $50 \%$ ) strictly prefer the median (by risk aversion).

For $A \geq E_{1}^{1}$ it is easily seen that

$$
E\left(F_{a A, a}^{x_{0}}\right) \geq a A-a E_{1}^{1} \geq 0 \text { for all } x_{0} \in[a A-a, a A+a] .
$$


And then it follows by risk aversion that all voters with $x_{0} \leq 0$ strictly prefer the median over the interval. Analogously it follows that if $A \leq-E_{1}^{1}$ then all voters with $x_{0} \geq 0$ prefer strictly the median over the interval.

Thus the only cases left are $A \in\left(0, E_{1}^{1}\right)$ and $A \in\left(-E_{1}^{1}, 0\right)$. Suppose $A \in$ $\left(0, E_{1}^{1}\right)$. Then it is straightforward to check that for each voter with

$$
x_{0} \in\left\{x \geq 0 \mid L_{i}(x)=-1\right\} \cup\left\{x<0 \mid L_{i}(x) \leq 0\right\}
$$

the mean of the voter's perception of the interval is further away from $x_{0}$ than the median (0). Thus all these voters strictly prefer the median over the interval (by risk aversion). Since the set above is the complement of $X_{i}^{+}$these voters constitute at least a weak majority. If $A \in\left(-E_{1}^{1}, 0\right)$ then it follows analogously that all voters with $x_{0} \notin X_{i}^{-}$strictly prefer the median over the interval. Again that is at least a weak majority.

Proof of Theorem 4.4.

1. First we show that

$$
\int_{-1}^{1} u_{x_{0}}(x) d F_{1}^{x_{0}}(x)
$$

is a continuous function of $x_{0}$ on $[-1,1]$. Let $x_{0} \in[-1,1]$ and et $\left(x_{n}\right)$ be a sequence in $[-1,1]$ such that $x_{n} \rightarrow x_{0}$. Then we have

$$
\begin{aligned}
& \left|\int_{-1}^{1} u_{x_{n}}(x) d F_{1}^{x_{n}}(x)-\int_{-1}^{1} u_{x_{0}}(x) d F_{1}^{x_{0}}(x)\right| \\
\leq & \left|\int_{-1}^{1} u_{x_{n}}(x) d F_{1}^{x_{n}}(x)-\int_{-1}^{1} u_{x_{0}}(x) d F_{1}^{x_{n}}(x)\right| \\
& +\left|\int_{-1}^{1} u_{x_{0}}(x) d F_{1}^{x_{n}}(x)-\int_{-1}^{1} u_{x_{0}}(x) d F_{1}^{x_{0}}(x)\right| \\
\leq & \max _{x \in[-1,1]}\left|u_{x_{n}}(x)-u_{x_{0}}(x)\right| \\
& +\left|\int_{-1}^{1} u_{x_{0}}(x) d F_{1}^{x_{n}}(x)-\int_{-1}^{1} u_{x_{0}}(x) d F_{1}^{x_{0}}(x)\right| .
\end{aligned}
$$

The first term in the last expression converges to zero because of the continuity of $u_{0}$ and the compactness of $[-1,1]$. The second term converges to zero by the continuity assumption on the $F_{1}^{x_{0}}$ 's. That proves the continuity of $\int_{-1}^{1} u_{x_{0}}(x) d F_{1}^{x_{0}}(x)$.

Then we can find a $0<C<E_{1}^{1}$ and an $\varepsilon>0$ such that

$$
\int_{-1}^{1} u_{x_{0}}(x) d F_{1}^{x_{0}}(x)>u_{x_{0}}(C) \text { for all } x_{0} \in[-1, \varepsilon] \text {. }
$$


The following two claims finishes the proof of the first part of the theorem.

Claim A: For sufficiently small $a>0,[-a, a]$ announced by Candidate 1 defeats any $y \in(-a C, a C)$ announced by Candidate 2 .

Proof: Choose an $N \in \mathbb{N}$ such that a strict majority of voters have preferred points in the set

$$
X_{1}^{N}=\left\{x\left|L_{1}(x)=1, \frac{1}{N} \leq\right| x \mid \leq N\right\} .
$$

It suffices to show that for $a$ sufficiently small all voters with $x_{0} \in X_{1}^{N}, x_{0}>0$ will prefer $[-a, a]$ announced by Candidate 1 over $a C$ announced by Candidate 2 . Since $0<C<E_{1}^{1}$ that follows by Taylor's theorem as in earlier proofs.

Claim B: For sufficiently small $a>0,[-a, a]$ announced by Candidate 1 defeats any $y \notin(-a C, a C)$ announced by Candidate 2 .

Proof: Let $a<\beta$. From the homogeneity (of degree $\alpha$ ) of $u_{0}$ and what we have shown above it follows that for any $x_{0} \in[-a, a \varepsilon]$,

$$
\begin{aligned}
\int_{-a}^{a} u_{x_{0}}(x) d F_{a}^{x_{0}}(x) & =\int_{-1}^{1} u_{x_{0}}(a x) d F_{1}^{x_{0}}(a x)=a^{\alpha} \int_{-1}^{1} u_{\frac{x_{0}}{a}}(x) d F_{1}^{\frac{x_{0}}{a}}(x) \\
& >a^{\alpha} u_{\frac{x_{0}}{a}}(C)=u_{x_{0}}(a C) .
\end{aligned}
$$

Thus we see that all voters with $x_{0} \in[-a, a \varepsilon]$ strictly prefer $[-a, a]$ announced by Candidate 1 over any certain position $y \geq a C$.

Voters with $x_{0} \in(-\beta,-a)$ has the same perception of $[-a, a]$ announced by Candidate 1 as voters with $x_{0}=-a$. And they are less (absolute) risk-averse on $[-a, a]$. Therefore these voters also prefer $[-a, a]$ announced by Candidate 1 over any $y \geq a C$.

By Taylor's theorem it is easily seen that voters with $x_{0}=-\beta$ and a neutral attitude towards Candidate 1 strictly prefer $[-a, a]$ announced by Candidate 1 over any $y \geq a C$ when $a$ is small enough. The same is true for voters with $x_{0}<-\beta$ because they are less risk averse on $[-a, a]$.

Thus all voters with $x_{0} \leq a \varepsilon$ strictly prefer $[-a, a]$ announced by Candidate 1 to any $y \geq a C$ for $a$ sufficiently small. By symmetry it follows that, for $a$ sufficiently small, all voters with $x_{0} \geq-a \varepsilon$ stricly prefer $[-a, a]$ announced by Candidate 1 to any $y \leq-a C$. That ends the proof of the claim.

2. Suppose Candidate 1 announces $[A-a, A+a]$ for some $A \geq 0$ (if $A<0$ the proof is analogous). Then, by the homogeneity of $u_{0}$ and the condition in the statement, we have that all voters with $x_{0} \in(A-a, A)$ strictly prefer $A-a E_{1}^{1}$ announced by Candidate 2 over the interval announced by Candidate 1 . By risk aversion the same is true for voters with $x_{0} \leq A-a$. Thus at least $50 \%$ of the voters strictly prefer $A-a E_{1}^{1}$ announced by Candidate 2 over the interval announced by Candidate 1 . 
Proof of Lemma 4.5.

Suppose

$$
\int_{-1}^{1} u_{0}(x) d F_{1}^{0}(x) \leq u_{0}\left(E_{1}^{1}\right)
$$

By the first assumption in the lemma it follows that, for all $0<x_{0}<1$,

$$
\int_{-1}^{1} u_{x_{0}}(x) d F_{1}^{x_{0}}(x) \leq \int_{-1}^{1} u_{0}(x) d F_{1}^{0}(x)
$$

Therefore we have that, for all $x_{0} \in\left(0, E_{1}^{1}\right]$,

$$
\int_{-1}^{1} u_{x_{0}}(x) d F_{1}^{x_{0}}(x) \leq \int_{-1}^{1} u_{0}(x) d F_{1}^{0}(x) \leq u_{0}\left(E_{1}^{1}\right)<u_{x_{0}}\left(E_{1}^{1}\right) .
$$

For $x_{0} \in\left(E_{1}^{1}, 1\right)$ it follows from the second assumption in the lemma that $E_{1}^{1}$ is (weakly) closer to $x_{0}$ than $E_{1}^{x_{0}}$. So by risk aversion it follows that, for all $x_{0} \in\left(E_{1}^{1}, 1\right)$,

$$
\int_{-1}^{1} u_{x_{0}}(x) d F_{1}^{x_{0}}(x)<u_{x_{0}}\left(E_{1}^{x_{0}}\right) \leq u_{x_{0}}\left(E_{1}^{1}\right) .
$$

Thus the condition in part 2. of Theorem 4.4 is satisfied.

Proof of Lemma 4.6.

For each $x_{0} \in[-1,0]$ let $C_{U}^{x_{0}}>x_{0}$ be defined by

$$
\frac{1}{2} \int_{-1}^{1} u_{x_{0}}(x) d x=u_{x_{0}}\left(C_{U}^{x_{0}}\right)
$$

If $C_{U}^{x_{0}} \leq C_{U}^{0}$ then we have

$$
\delta u_{x_{0}}(0)+(1-\delta) u_{x_{0}}\left(C_{U}^{0}\right) \leq \delta u_{x_{0}}\left(x_{0}\right)+(1-\delta) u_{x_{0}}\left(C_{U}^{x_{0}}\right)=u_{x_{0}}\left(C^{x_{0}}\right) .
$$

So the certainty equivalent (the one to the right of the preferred point) of the lottery "0 with probability $\delta, C_{U}^{0}$ with probability $1-\delta$ " for a voter at $x_{0}$ is greater than or equal to $C^{x_{0}}$. The certainty equivalent of the same lottery for a voter at 0 is $C^{0}$. And since voters at $x_{0}$ are less absolute risk averse on $\left[0, C_{U}^{0}\right]$ than voters at 0 the certainty equivalent for a voter at 0 is greater than that for a voter at $x_{0}$. Thus we must have $C^{x_{0}} \leq C^{0}$. So we see that it suffices to show that $C_{U}^{x_{0}} \leq C_{U}^{0}$ for all $x_{0} \in[-1,0)$. 
Let $x_{0} \in[-1,0)$. Define $C_{U-}^{x_{0}}, C_{U+}^{x_{0}}>x_{0}$ and $C_{U-}^{0}, C_{U+}^{0}>0$ by

$$
\frac{1}{2+x_{0}} \int_{-1}^{x_{0}+1} u_{x_{0}}(x) d x=u_{x_{0}}\left(C_{U-}^{x_{0}}\right), \quad \frac{1}{-x_{0}} \int_{x_{0}+1}^{1} u_{x_{0}}(x) d x=u_{x_{0}}\left(C_{U+}^{x_{0}}\right)
$$

and

$$
\frac{1}{2+x_{0}} \int_{-1}^{x_{0}+1} u_{0}(x) d x=u_{0}\left(C_{U-}^{0}\right), \quad \frac{1}{-x_{0}} \int_{x_{0}+1}^{1} u_{0}(x) d x=u_{0}\left(C_{U+}^{0}\right) .
$$

It is easily seen that

$$
C_{U-}^{x_{0}} \leq C_{U-}^{0} \text { and } C_{U+}^{x_{0}} \leq C_{U+}^{0}
$$

Thus we have that

$$
\frac{2+x_{0}}{2} u_{x_{0}}\left(C_{U-}^{0}\right)+\frac{-x_{0}}{2} u_{x_{0}}\left(C_{U+}^{0}\right) \leq \frac{2+x_{0}}{2} u_{x_{0}}\left(C_{U-}^{x_{0}}\right)+\frac{-x_{0}}{2} u_{x_{0}}\left(C_{U+}^{x_{0}}\right)=u_{x_{0}}\left(C_{U}^{x_{0}}\right) .
$$

So the certainty equivalent (the one to the right of the preferred point) of the lottery " $C_{U-}^{0}$ with probability $\frac{2+x_{0}}{2}, C_{U+}^{0}$ with probability $\frac{-x_{0}}{2}$ " for a voter at $x_{0}$ is greater than or equal to $C_{U}^{x_{0}}$. The certainty equivalent of the same lottery for a voter at 0 is $C_{U}^{0}$. And since voters at $x_{0}$ are less absolute risk averse on $\left[C_{U-}^{0}, C_{U+}^{0}\right]$ than voters at 0 the certainty equivalent for a voter at 0 is greater than that for a voter at $x_{0}$. Thus we must have $C_{U}^{x_{0}} \leq C_{U}^{0}$.

\section{Proof of Lemma 4.7.}

For each $x_{0} \in[-1,0]$ let $C_{U}^{x_{0}}>x_{0}$ be defined an in the proof of lemma 4.6. Furthermore let $0<D^{0}<C_{U}^{0}$ be defined by

$$
\int_{-\frac{1}{2}}^{\frac{1}{2}} u_{0}(x) d x=u_{0}\left(D^{0}\right)
$$

From the proof of Lemma 4.6 we know that $C_{U}^{x_{0}} \leq C_{U}^{0}$ for all $x_{0} \in[-1,0]$. Therefore we have, for each $x_{0} \in\left[-\frac{1}{2}, 0\right]$,

$$
\gamma u_{x_{0}}\left(D^{0}\right)+(1-\gamma) u_{x_{0}}\left(C_{U}^{0}\right) \leq \gamma \int_{x_{0}-\frac{1}{2}}^{x_{0+\frac{1}{2}}} u_{x_{0}}(x) d x+(1-\gamma) u_{x_{0}}\left(C_{U}^{x_{0}}\right)=u_{x_{0}}\left(C^{x_{0}}\right)
$$

So the certainty equivalent (the one to the right of the preferred point) of the lottery " 0 with probability $\gamma, C_{U}^{0}$ with probability $1-\gamma$ " for a voter at $x_{0}$ is greater than or equal to $C^{x_{0}}$. The certainty of the same lottery for a voter at 0 is $C^{0}$. And since voters at $x_{0}$ are less risk averse on $\left[D^{0}, C_{U}^{0}\right]$ than voters at 0 the certainty 
equivalent for a voter at 0 is greater than that for a voter at $x_{0}$. Thus we must have $C^{x_{0}} \leq C^{0}$.

Proof of Lemma 4.8.

For each $x_{0} \in\left[-1,-\frac{1}{2}\right]$ the assimilation perception of $[-1,1]$ is a convex combination of the uniform distribution on $[-1,0]$ and the uniform distribution on $[-1,1]$. Let $C_{U}^{x_{0}}$ be defined as in the proof of lemma 4.6. Furthermore let $D^{x_{0}}>x_{0}$ be defined by

$$
\int_{-1}^{0} u_{x_{0}}(x) d x=u_{x_{0}}\left(D^{x_{0}}\right)
$$

Mimicking arguments from the proof of Lemma 4.6 it follows that $C_{U}^{x_{0}} \leq C_{U}^{-\frac{1}{2}}$ and $D^{x_{0}} \leq D^{-\frac{1}{2}}$ for all $x_{0} \in\left[-1,-\frac{1}{2}\right]$. Therefore we have that for each $x_{0} \in\left[-1,-\frac{1}{2}\right]$,

$$
\gamma u_{x_{0}}\left(D^{-\frac{1}{2}}\right)+(1-\gamma) u_{x_{0}}\left(C_{U}^{-\frac{1}{2}}\right) \leq \gamma u_{x_{0}}\left(D^{x_{0}}\right)+(1-\gamma) u_{x_{0}}\left(C_{U}^{x_{0}}\right)=u_{x_{0}}\left(C^{x_{0}}\right)
$$

And since a voter at $x_{0}<-\frac{1}{2}$ is less risk averse than a voter at $-\frac{1}{2}$ on $\left[D^{-\frac{1}{2}}, C_{U}^{-\frac{1}{2}}\right]$ it follows that $C^{x_{0}} \leq C^{-\frac{1}{2}}$ for all $x_{0} \in\left[-1,-\frac{1}{2}\right]$ (same argument as in the two previous proofs).

Proof of Theorem 4.9.

Suppose $\left(\Delta_{1}, \Delta_{2}\right)$ is a mixed strategy Nash equilibrium. We will show that this leads to a contradiction.

Let $\varepsilon>0$. Then there exists $a^{\prime}>0$ such that $\Delta_{2}$ puts less than $\varepsilon$ probability mass on the certain positions in $\left(-a^{\prime}, a^{\prime}\right) \backslash\{0\}$. Therefore, by announcing the ambiguous position $[-a, a]$ for $a$ sufficiently close to zero Candidate 1 can defeat $\Delta_{2}$ with probability greater than $1-\varepsilon$ (Candidate 1 defeats all certain positions not in $\left.\left(-a^{\prime}, a^{\prime}\right) \backslash\{0\}\right)$. Thus it follows that in the equilibrium $\left(\Delta_{1}, \Delta_{2}\right)$, Candidate 1 must win with probability one.

Pick $\bar{A} \in \mathbb{R}, \bar{a}>0$ such that, for any neighborhood $B$ of $(\bar{A}, \bar{a}), \Delta_{1}$ puts positive probability on

$$
\{[A-a, A+a] \mid(A, a) \in B\} .
$$

The following claim shows that for some $B$ Candidate 2 has a position that defeats all positions in the set above. Thus it follows that in the equilibrium $\left(\Delta_{1}, \Delta_{2}\right)$, Candidate 2 must win with some positive probability. That is a contradiction. 
Claim: Suppose Candidate 2 announces

$$
\left\{\begin{array}{c}
\bar{A}+\bar{a} E_{1}^{1} \text { if } \bar{A} \leq 0 \\
\bar{A}-\bar{a} E_{1}^{1} \text { if } \bar{A}>0
\end{array}\right\} .
$$

Then there exists some neighborhood $B$ of $(\bar{A}, \bar{a})$ such that he defeats all positions of Candidate 1 in the set

$$
\{[A-a, A+a] \mid(A, a) \in B\} .
$$

Proof: We will only do the proof for $\bar{A} \leq 0$, the other case is completely analogous. By announcing $\bar{A}+\bar{a} E_{1}^{1}$ Candidate 2 defeats $[\bar{A}-\bar{a}, \bar{A}+\bar{a}]$ (by Theorem 4.4, part 2.). We have to show that he also defeats "nearby" ambiguous positions. Suppose not. Then there exists a sequence $\left(A_{n}, a_{n}\right)$ that converges to $(\bar{A}, \bar{a})$ and satisfies that, for any $n,\left[A_{n}-a_{n}, A_{n}+a_{n}\right]$ announced by Candidate 1 gets at least a tie against $\bar{A}+\bar{a} E_{1}^{1}$ announced by Candidate 2 . Therefore, there must exist $x_{0}$ 's such that

$$
\int u_{x_{0}}(x) d F_{A_{n}, a_{n}}^{x_{0}}(x) \nrightarrow \int u_{x_{0}}(x) d F_{\bar{A}, \bar{a}}^{x_{0}}(x) .
$$

(If $x_{0}<A_{n}-a_{n}$ then $F_{A_{n}, a_{n}}^{x_{0}}$ means $F_{A_{n}, a_{n}}^{A_{n}-a_{n}}$ and so on). By using the definitions of $F_{A_{n}, a_{n}}^{x_{0}}$ and $F_{\bar{A}, \bar{a}}^{x_{0}}$ and the continuity assumption on the $F_{1}^{x_{0}}$ 's in the section on winning strategies it follows that there is convergence for all $x_{0}$ 's. Thus we have a contradiction. 